Supplementary Information for

\title{
Strong polarity asymmetry and abnormal mechanical electroresistance effect in organic monolayer tunnel junction
}

Gelei Jiang $^{1,2}$, Yun Chen ${ }^{1,2}$, Ye Ji ${ }^{1,2}$, Weijin Chen ${ }^{1,2,3^{*}}$, Xiaoyue Zhang ${ }^{1,2,4^{*}}$, Yue Zheng $^{1,2 *}$

${ }^{1}$ State Key Laboratory of Optoelectronic Materials and Technologies, School of Physics, Sun Yatsen University, Guangzhou 510275, China.

${ }^{2}$ Micro and Nano Physics and Mechanics Research Laboratory, School of Physics, Sun Yat-sen University, Guangzhou 510275, China.

${ }^{3}$ School of Materials, Sun Yat-sen University, Guangzhou 510275, China.

${ }^{4}$ Sino-French Institute of Nuclear Engineering and Technology, Sun Yat-sen University, Zhuhai, 519082, China.

\footnotetext{
AUTHOR INFORMATION

Corresponding Authors

*E-mail: zhengy35@mail.sysu.edu.cn (Y.Z.).

*E-mail: chenweijin@ mail.sysu.edu.cn (W.J.C).

*E-mail: zhangxy26@mail.sysu.edu.cn (X.Y.Z).
} 
(a)

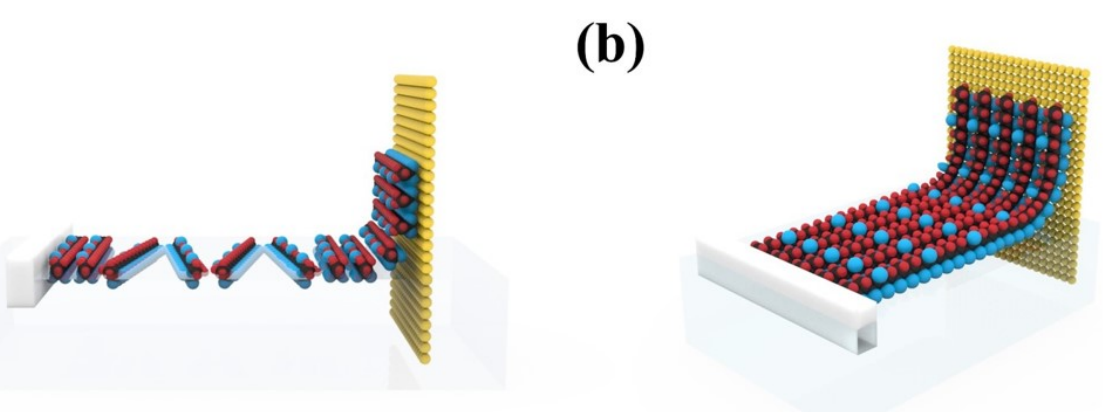

(c)

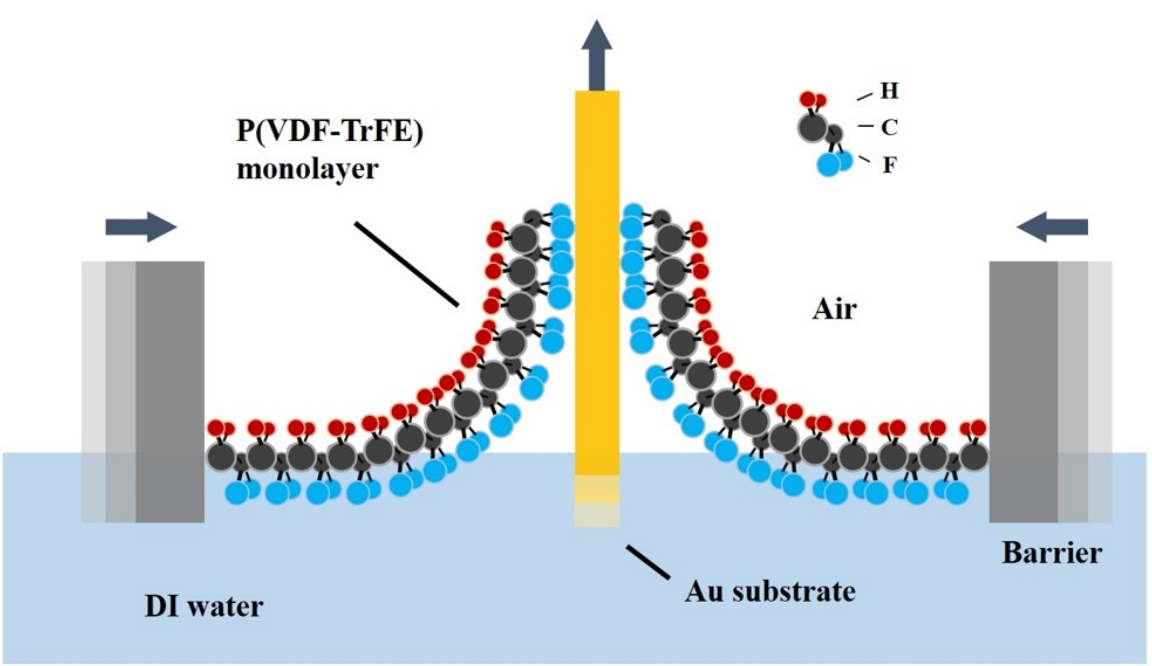

Figure S1. Schematic of the preparation process of $\mathrm{P}(\mathrm{VDF}-\mathrm{TrFE})$ monolayer via the LB technique. (a) During the preparation process, $\mathrm{P}(\mathrm{VDF}-\mathrm{TrFE})$ molecular chains in the polymer solvent were dispersed and arranged on the surface of deionized water in the LB trough. (b) Single monolayer of P(VDF-TrFE) was transferred onto the Au (111) surface at a surface pressure of $5 \mathrm{mN} \mathrm{m}^{-1}$ at room temperature. (c) 2D front view of the transfer process. 
Surface pressure versus Trough area

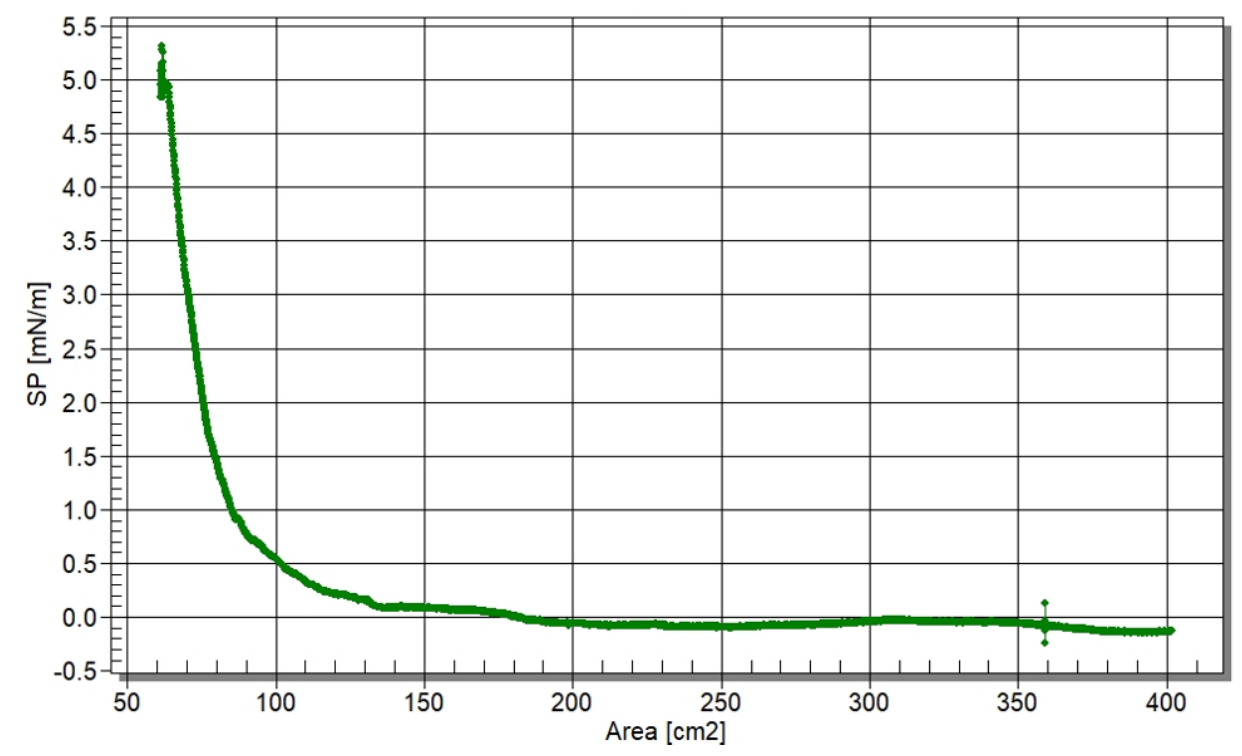

Figure S2. The pressure-area isotherm of $\mathrm{P}(\mathrm{VDF}-\mathrm{TrFE})$ at room temperature during preparation. 


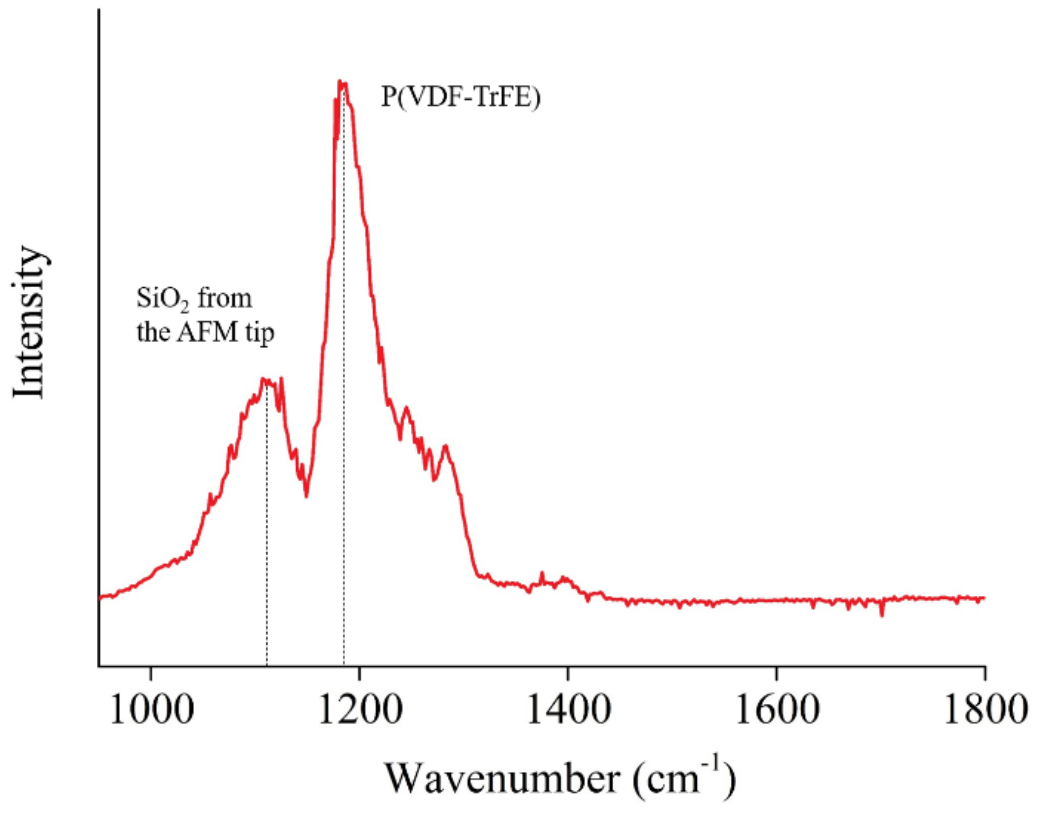

Figure S3. The IR spectra of P(VDF-TrFE) monolayer measured with a nanoIR2-FS by Anasys Instruments under ambient condition. 
(a)

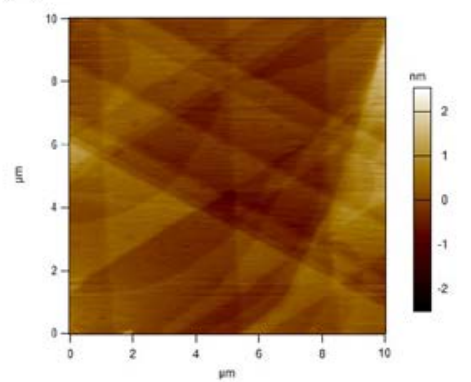

(d)

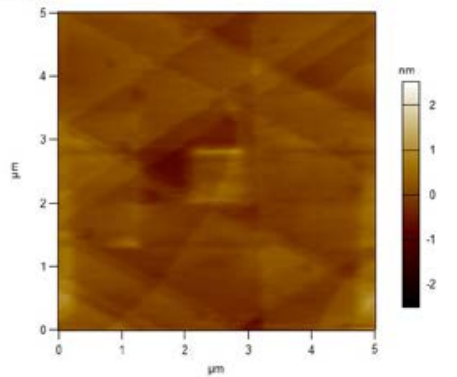

(b)

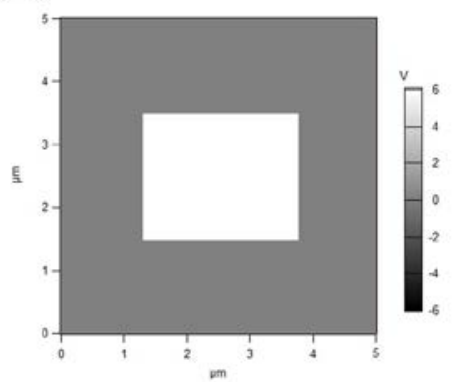

(e)

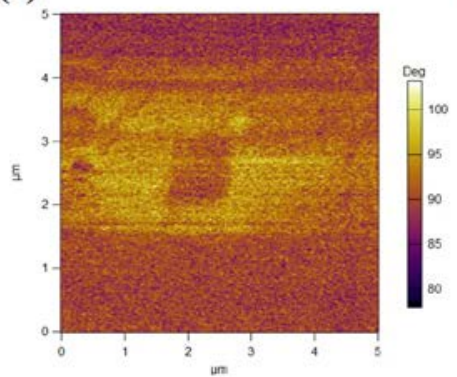

(c)

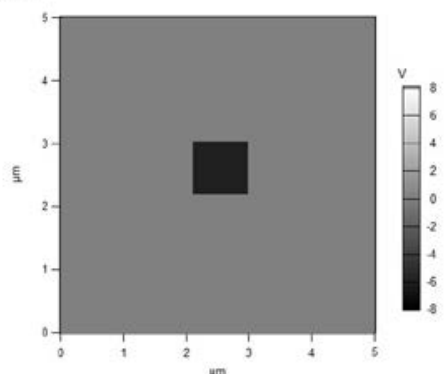

(f)

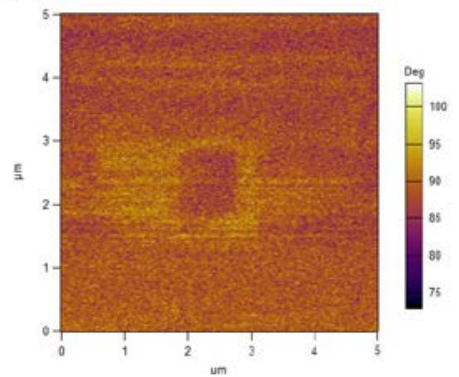

Figure S4. PFM characterization of domain pattern in the P(VDF-TrFE) monolayer. A $2 \times 2 \mu \mathrm{m}^{2}$ square area (b) was first written with a positive voltage (applied to the PFM tip); then a $1 \times 1 \mu \mathrm{m}^{2}$ square area (c) was written with a negative voltage. The topography images (a) before and (d) after writing show that the sample is not damaged during the writing process. The phase contrast of the writing domain pattern (e) is rather weak due to the strong polarity asymmetry of the P(VDF-TrFE) monolayer. During the PFM scanning process, the written downward polarity state ("H-Au" state) cannot maintain stable and relaxes back to the upward polarity state ("F-Au" state). The phase contrast is even weaker after a second scanning (f). 


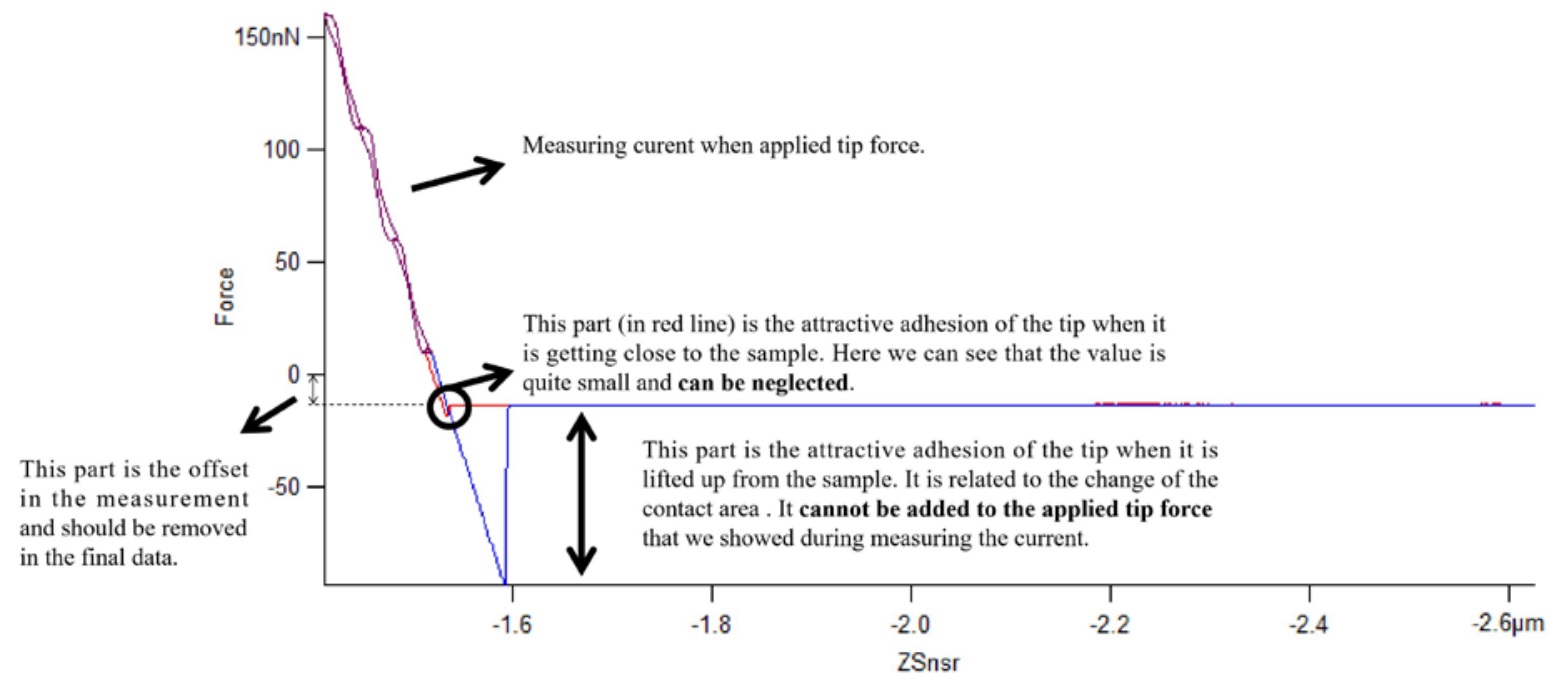

Figure S5. The raw data of the force curve during measuring the results in Fig. 5(a). 
(a)

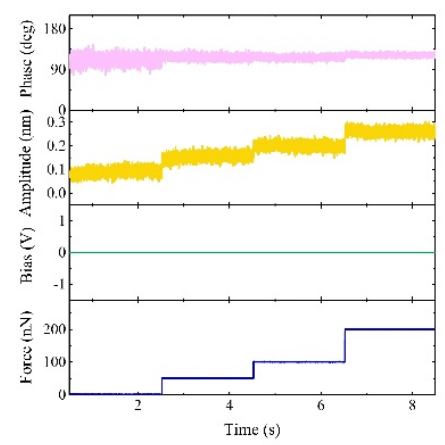

(b)

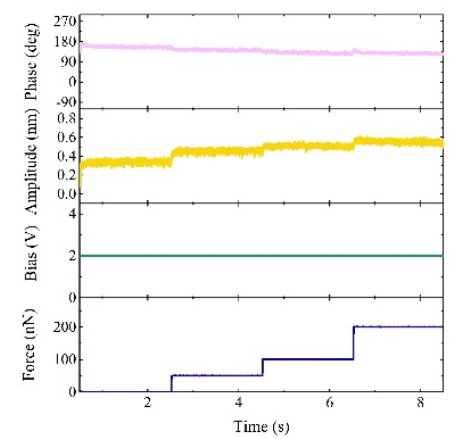

(c)

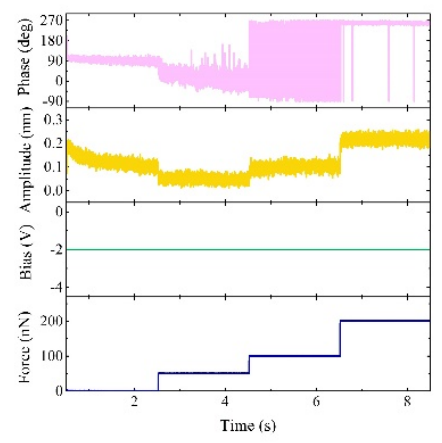

Figure S6. The change of PFM phase and amplitude of P(VDF-TrFE) monolayer under a time-increasing tip force ( $0 \mathrm{nN} \sim 200 \mathrm{nN}$ ) and at (a) zero, (b) $2 \mathrm{~V}$ or (c) $-2 \mathrm{~V}$ constant biases. 


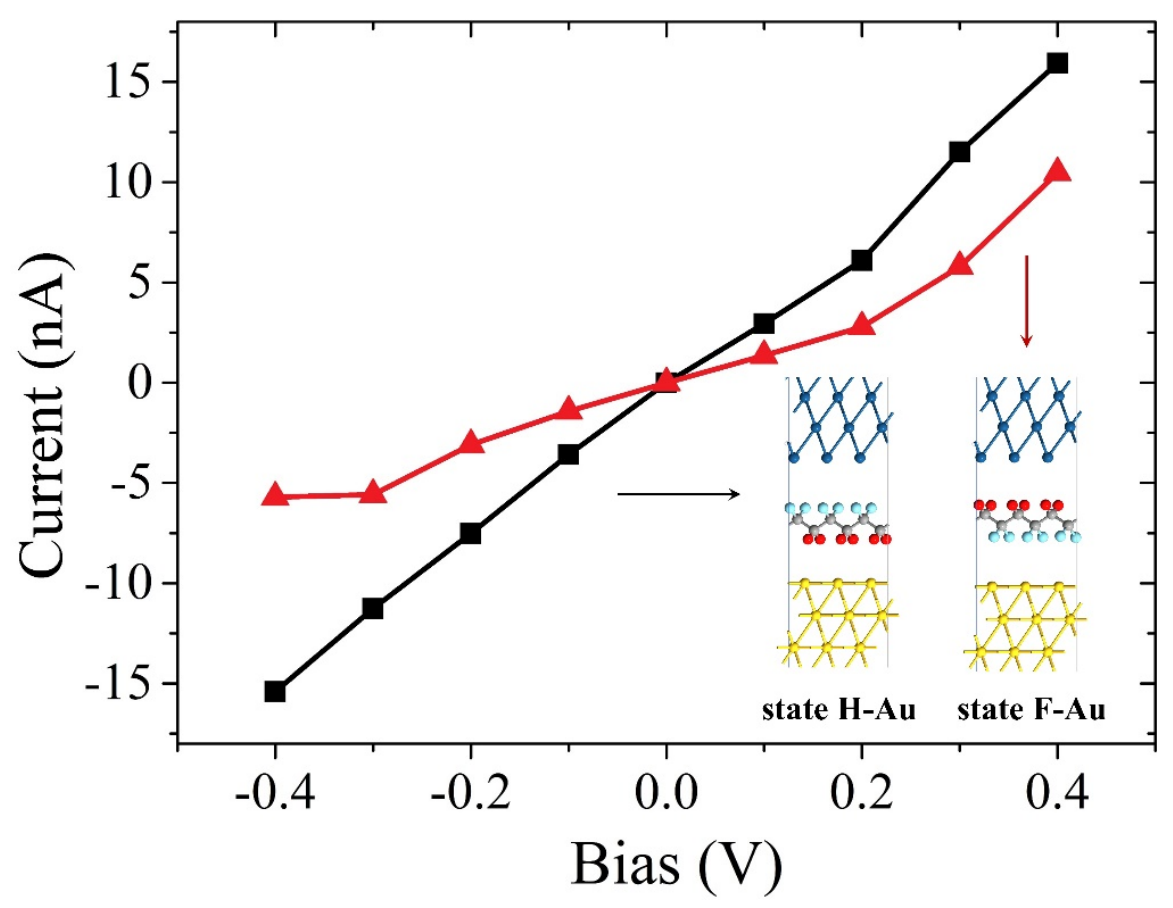

Figure S7. Current-voltage curves of the two states ("F-Au" and "H-Au" state) of PVDF monolayer predicted by density functional theory (DFT) calculations.

The equilibrium interface distances were determined for the $\mathrm{H} / \mathrm{Au}$ and $\mathrm{F} / \mathrm{Au}$ interfaces respectively, after structural relaxation performed in the Vienna Ab initio Simulation Package (VASP) under periodic boundary conditions. A plane-wave basis set and projector augmented wave (PAW) potentials are employed. The exchange correlation potential is treated in the local density approximation (LDA), and the plane wave functions are expanded with an energy cutoff of $500 \mathrm{eV}$. The dispersion-corrected density functional theory (DFT-D) is considered, in which the Van der Walls interactions are included semiempirically. All the atoms are relaxed until the HellmannFeynman force on each atom is less than $10 \mathrm{meV}^{-1}$.

The electron transport properties of the PVDF monolayer was calculated by the Atomistix ToolKit (ATK) based on nonequilibrium Green's function (NEGF) in combination with DFT. The above relaxed structures were used to construct the scattering region, and the periodicity of the supercell in the transport directions was replaced by a matching of two semi-infinite electrodes. The valence electrons are expanded in a numerical atomic-orbital basis set of single zeta plus polarization (SZP) basis. To get converged results of the conductance, we used a dense k-point mesh of $10 \times 10$ to sample in the surface two-dimensional Brillouin zone for the two-probe system. 
(a)

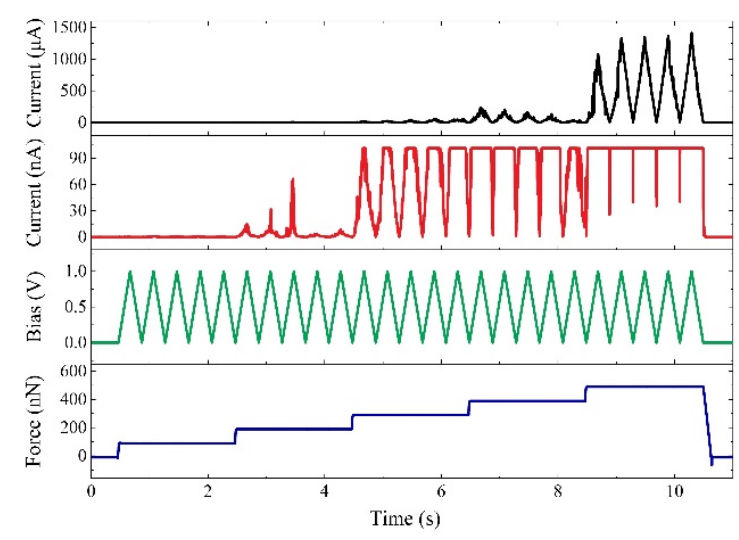

(b)

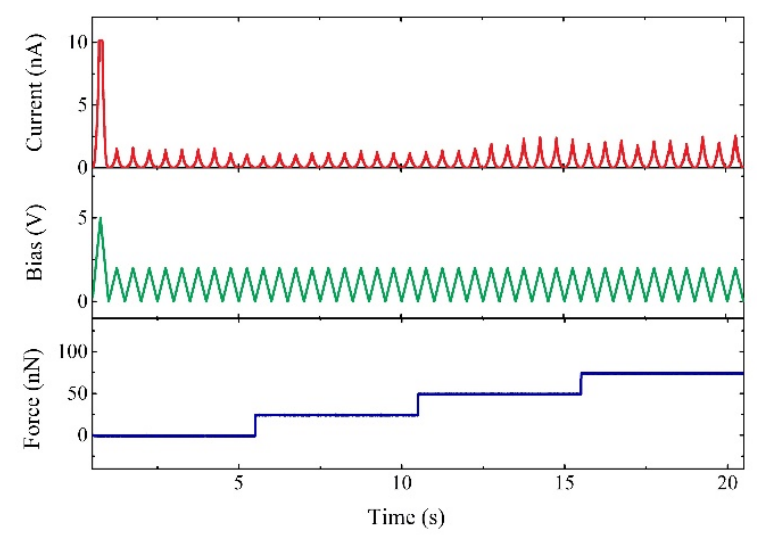

Figure S8. (a) Current change of the P(VDF-TrFE) monolayer under an increasing tip force ( $0 \mathrm{nN} \sim 500 \mathrm{nN}$ ). No preset bias voltage was applied. (b) Current change of the $\mathrm{P}(\mathrm{VDF}-\mathrm{TrFE})$ monolayer under an increasing tip force $(0 \mathrm{nN} \sim 50 \mathrm{nN})$. A positive preset bias voltage (+write) was applied at the beginning. 


\begin{tabular}{|c|c|c|c|c|}
\hline Structure & Writing bias & Resistance state & TOTEN $(\mathrm{eV})$ & $\begin{array}{c}\text { Interface } \\
\text { distance }(\AA)\end{array}$ \\
\hline "F-Au" & Positive & OFF & -1364.08 & 3.2 \\
\hline "H-Au" & Negative & ON & -1363.92 & 2.8 \\
\hline
\end{tabular}

Table S1. The writing bias, resistance state, DFT calculated total energy, and interface distance of the F-Au and H-Au interfaces of PVDF monolayer in "F-Au" state and "H-Au” state. 